\title{
Immunotherapy Targeted at Brain Cancer Stem Cells
}

\author{
Masahiro Toda* \\ Department of Neurosurgery, Keio University School of Medicine, 35 Shinanomachi, Shinjuku-ku, Tokyo 160-8582, \\ Japan
}

\begin{abstract}
Progress in stem cell research has been associated with the discovery of the presence of cancer stem cells in a variety of malignant tumors, and because these cells are resistant to anticancer agents and radiotherapy, analysis of their properties has been rapidly pursued as an important target for the treatment of cancers, including malignant brain tumors. Brain cancer stem cells (BCSCs) have been isolated from brain tumor tissue and brain tumor cell lines by using neural stem cell culture methods. Analyzing the properties of BCSCs is extremely important to developing treatment methods that target BCSCs. Although no treatment method targeting BCSCs has yet been established, several methods have been proposed based on their cell-biological characteristics. This article describes therapeutic strategies that target drug-resistance molecules and stem-cell-associated molecules as well as immunotherapy targeted at BCSCs.
\end{abstract}

Key Words: Cancer stem cell, glioma, immunotherapy, vaccine.

\section{DRUG RESISTANCE MOLECULES}

Genetic mutations in the target molecules of drugs, expression of molecules that inactivate drugs, and mechanisms that excrete drugs from cells have all been identified as ways that cancer cells acquire resistance to chemotherapeutic agents [1]. Some tumors that initially respond to chemotherapy subsequently recur, and the tumor cells in such cases were considered to have acquired drug resistance. After the existence of cancer stem cells was demonstrated, however, and because cancer stem cells are proficient at DNA repair and exhibit high gene expression levels of ATP-binding cassette half-transporter proteins ( $\mathrm{ABC}$ transporters), the following new hypothesis was proposed: cancer stem cells are resistant to anticancer agents, and they persist after chemotherapy and become the source of tumor regrowth [1]. Several reports have described expression of multidrug resistance genes in glioblastoma-derived BCSCs [2] and resistance of BCSCs to the anticancer drug BCNU [3].

Inhibitors of $\mathrm{ABC}$ transporters, which are drug resistance molecules, have attracted interest as candidate therapeutic agents for use against cancer stem cells. Although drugs targeting $\mathrm{ABC}$ transporters have already been developed, they have primarily been targeted against multidrug resistance (MDR)-1, encoded by the ABCB1 gene. Because of the high levels of ABCG2 and ABCG5 expression in cancer stem cells, inhibitors and antibodies to these molecules are being considered as candidate therapeutic agents. GF120918 [4] and XR9576 [5] are drugs that inhibit ABCG2 as well as $\mathrm{ABCB} 1$, and clinical trials are currently being planned. Re-

*Address correspondence to this author at the Department of Neurosurgery, Keio University School of Medicine, 35 Shinanomachi, Shinjuku-ku, Tokyo 160-8582, Japan; Tel: 3-5363-3808; Fax: 3-3354-8053;

E-mail: todam@sc.itc.keio.ac.jp cently, an antibody against ABCG5 has been reported to inhibit tumorigenesis by cancer stem cells [6], and a more detailed analysis of its therapeutic effect is eagerly awaited.

\section{STEM-CELL-ASSOCIATED MOLECULES}

Hedgehog (Shh) and Notch signals are important for maintaining the stemness of stem cells, and their signals are involved in carcinogenesis. Recently, their roles in cancer stem cells have attracted interest. Hedgehog $(\mathrm{HH})$ binds to the cell surface receptor Patched (PTCH) and transmits a signal to the Smoothened (SMO) and GLI proteins. The SMO inhibitor cyclopamine has been shown to inhibit BCSC proliferation and tumorigenesis $[7,8]$. A Notch signal inhibitor, $\gamma$-secretase inhibitor, has also been shown to inhibit both proliferation by BCSCs and their tumor-forming ability [9].

An attempt to eliminate BCSCs with bone morphogenetic protein (BMP), a molecule that induces the differentiation of neural stem cells (NSCs) into astrocytes, has been reported [10]. Since BMP4 exhibits an antitumor effect against transplanted tumors in vivo, the development of methods for targeting BCSCs with molecules that induce stem cell differentiation has attracted much interest. The microenvironment for maintaining stem cells has also been suggested to be important for the maintenance of BCSCs [11]. Vascular endothelial growth factor (VEGF), an endothelial growth factor, has been shown to control the BCSC microenvironment, and because VEGF antibodies have already been applied clinically, VEGF antibodies are expected to serve as a therapeutic agent against BCSCs.

\section{IMMUNOTHERAPY}

Immunotherapy consists of active immunotherapy, which induces and strengthens antitumor immunity in cancer patients' own bodies by vaccinating patients with tumor cells 
themselves or identified tumor antigens, and passive immunotherapy, in which cells that are important to tumor immunity, such as cytotoxic T lymphocytes (CTLs) and natural killer (NK) cells, are prepared in vitro and administered to patients. Since tumor antigens have now been identified [12], tumor vaccine therapy, which consists of active immunotherapy that induces tumor-specific immunity, is expected to have some degree of efficacy.

The brain is an immune-privileged organ, and because dendritic cells and other antigen-presenting cells do not function adequately, it is difficult to present brain tumor antigens to the immune system [13]. However, since activated T cells have been found to pass through the blood-brain barrier (BBB) and to infiltrate the brain [14], a treatment that destroys brain tumors by subcutaneously injecting a tumor vaccine and inducing systemic immunity against brain tumors is being attempted. Several clinical trials of a tumor vaccine therapy for gliomas have been conducted $[15,16]$. Steiner et al reported an improvement of the prognosis of glioblastoma patients by vaccination with virus-modified autologous tumor cells [15]. Yu et al. also reported a therapeutic effect in some of the cases without any severe adverse effects [16]. While the efficacy of these vaccine therapies should be determined in further randomized clinical trials, no marked adverse effects have been observed, thereby demonstrating the safety of tumor vaccine therapy for brain tumors.

If BCSC tumor antigens can be identified, it might become possible to develop tumor-specific immunotherapies. Immunotherapy with BCSCs themselves, rather than with tumor antigens, may also be a feasible method of treating brain tumors.

\section{IMMUNOTHERAPY WITH TUMOR ANTIGENS}

As a result of the discovery of tumor antigen peptides that are recognized by $\mathrm{T}$ cells, it has become possible to artificially manipulate the immune response as a target of immunotherapy, and that has allowed great advances to be made in tumor immunology [17]. Moreover, because T cells have been demonstrated to be important for tumor rejection, to have the ability to proliferate tumor-specifically, and to have a memory mechanism, they play a central role in tumor vaccine therapy. Major histocompatibility complex (MHC) class I binding antigen peptides, which are recognized by CTLs (CD8-positive $\mathrm{T}$ cells), and MHC class II binding peptides, which are recognized by helper $\mathrm{T}$ cells (CD4-positive $\mathrm{T}$ cells), are among the tumor antigens recognized by $\mathrm{T}$ cells [12]. Because of technical reasons related to the identification of peptides, MHC class I binding antigen peptides with the ability to activate CTLs have mainly been used in the preparation of tumor vaccines.

The tumor antigens recognized by CTLs thus far have been classified into three representative categories [12]. The cancer-testis (CT) category consists of antigens that are expressed in various cancer tissues as well as in normal testis, ovary, and placenta. Because expression of MHC molecules by reproductive system cells is very low, CTLs against CT antigens selectively attack only the cancer cells and do not attack the reproductive cells, thereby making CT antigens ideal target antigens. The tissue-specific category consists of tumor antigens that are also expressed in the normal tissue from which the tumor originates but that are expressed at higher levels in tumor cells and are recognized by CTLs. The mutant antigen category consists of antigens that result from tumor-specific gene mutations and are recognized by CTLs as tumor antigens.

Vaccine therapy with tumor antigens can be administered by various techniques and by using various types of antigens, e.g., peptides, proteins, and genes, or in combination with adjuvants. Because peptides are biologically stable and easy to handle, clinical studies that have assessed antigen peptides represent the majority of research on the immunotherapy of brain tumors [18, 19]. We have been identifying and analyzing glioma antigens by a variety of techniques [20-22], and we consider SOX6, which was identified by using the SEREX (serological identification of antigens by recombinant expression cloning) method and belongs to the CT antigen category because of its expression specificity, to be an ideal target for treating gliomas [23]. We recently identified human leukocyte antigen (HLA)-A2- and A24-restricted CTL peptides derived from SOX6, and stimulation of glioma patients' lymphocytes with them revealed that the stimulated lymphocytes are capable of inducing glioma-specific CTLs [24]. We also demonstrated that BCSCs isolated from glioma patient specimens express SOX6 and are killed by CTLs primed against SOX6-derived peptides [24].

The identification of BCSC-derived antigen peptides that are recognized by CTLs has made clinical application of immunotherapy targeting BCSCs possible, and since the mechanism of the cytotoxicity differs from that of other treatment methods, it shows promise of becoming a component of multimodal therapy in the future.

\section{IMMUNOTHERAPY WITH BRAIN CANCER STEM CELLS}

The presence of tumor antigens has been demonstrated in tumor cells, but because tumor cells have very weak antigenicity, simply immunizing with tumor cells themselves does not induce adequate anti-tumor immunity. Because of this, tumor cells/tissues have been used to create tumor vaccines and modified to increase the immunogenicity of the tumor antigens [19]. Historically, haptens and various viruses have been used as adjuvants, and recent clinical trials of tumor vaccines have been conducted by administering the vaccine together with cytokines, such as granulocyte-macrophage colony-stimulating factor (GM-CSF), IL-2, IL-4, and IL-12, and with the costimulatory factor CD80. Tumor vaccine therapy in which tumor-cell-derived proteins (or peptides) are pulsed to dendritic cells has also been performed [19].

Clinical studies of tumor vaccines that contain IL-2 and IL-4 have been conducted as a treatment for gliomas [25, 26]. IL-2 genes were transduced into cultured glioma cells established from tumor tissue samples, and after irradiating the cells, the patients were subcutaneously inoculated with them as a tumor vaccine, and tumor regression was observed in some cases [26]. Because establishing cultured glioma cells from the resected tissue was often difficult, tumor vaccine therapy has also been performed by transducing IL-4 genes into patient-derived fibroblasts and inoculating pa- 
tients with a mixture of the fibroblasts and glioma cells and dendritic cells [25] .

The fact that these methods do not require the identification of tumor antigens and that, in theory, immunization with a variety of tumor antigens is possible, is a great advantage. The efficacy of individual antigens as tumor vaccines needs to be further assessed in the future, because the amounts of the individual antigens are very small and immunosuppressive components may be included. However, by isolating and concentrating BCSCs, vaccination with BCSCs could be applied clinically at an early stage, and thus it represents a promising treatment method.

\section{CONCLUSION}

In view of the absence of lymphatic organs in the brain and the presence of the $\mathrm{BBB}$, the brain is regarded as an immunologically privileged site. Based on the results of recent research, however, immunity can be modulated and controlled in the brain, similar to the situation in other organs. While the BBB protects against penetration of antibodies into the brain, activated $\mathrm{T}$ cells are capable of entering the brain across the BBB and selectively attacking BCSC targets. Thus, immunotherapy that induces cellular immunity shows promise of being an effective method of targeting BCSCs. It will also be important to develop multimodal treatment methods that use combinations of agents with different cytotoxic mechanisms to target BCSCs.

\section{REFERENCES}

[1] Dean M, Fojo T, Bates S. Tumour stem cells and drug resistance. Nat Rev Cancer 2005; 5: 275-84.

[2] Salmaggi A, Boiardi A, Gelati M, et al. Glioblastoma-derived tumorospheres identify a population of tumor stem-like cells with angiogenic potential and enhanced multidrug resistance phenotype. Glia 2006; 54: 850-60.

[3] Kang MK, Kang SK. Tumorigenesis of chemotherapeutic drug-resistant cancer stem-like cells in brain glioma. Stem Cells Dev 2007; 16: 837-47

[4] Sparreboom A, Planting AS, Jewell RC, et al. Clinical pharmacokinetics of doxorubicin in combination with GF120918, a potent inhibitor of MDR1 P-glycoprotein. Anticancer Drugs 1999; 10: 719-28.

[5] Agrawal M, Abraham J, Balis FM, et al. Increased $99 \mathrm{mTc}$-sestamibi accumulation in normal liver and drug-resistant tumors after the administration of the glycoprotein inhibitor, XR9576. Clin Cancer Res 2003; 9: 650-6.

[6] Schatton T, Murphy GF, Frank NY, et al. Identification of cells initiating human melanomas. Nature 2008; 451:345-9.

[7] Bar EE, Chaudhry A, Lin A, et al. Cyclopamine-mediated hedgehog pathway inhibition depletes stem-like cancer cells in glioblastoma. Stem Cells 2007; 25: 2524-33.
[8] Clement V, Sanchez P, de Tribolet N, Radovanovic I, Ruiz i Altaba A. HEDGEHOG-GLI1 signaling regulates human glioma growth: cancer stem cell self-renewal, and tumorigenicity. Curr Biol 2007; 17: $165-72$.

[9] Fan X, Matsui W, Khaki L, et al. Notch pathway inhibition depletes stem-like cells and blocks engraftment in embryonal brain tumors. Cancer Res 2006; 66: 7445-52.

[10] Piccirillo SG, Reynolds BA, Zanetti N, et al. Bone morphogenetic proteins inhibit the tumorigenic potential of human brain tumour-initiating cells. Nature 2006; 444: 761-5.

[11] Calabrese C, Poppleton H, Kocak M, et al. A perivascular niche for brain tumor stem cells. Cancer Cell 2007; 11: 69-82.

[12] Kawakami Y, Rosenberg SA. Human tumor antigens recognized by T-cells. Immunol Res 1997; 16: 313-39.

[13] Toda M. Immuno-viral therapy as a new approach for the treatment of brain tumors. Drug News Perspect 2003; 16: 223-9.

[14] Becher B, Prat A, Antel JP. Brain-immune connection: immuno-regulatory properties of CNS-resident cells. Glia 2000; 29: 293-304.

[15] Steiner HH, Bonsanto MM, Beckhove P, et al. Antitumor vaccination of patients with glioblastoma multiforme: a pilot study to assess feasibility, safety, and clinical benefit. J Clin Oncol 2004; 22: 4272-81.

[16] Yu JS, Wheeler CJ, Zeltzer PM, et al. Vaccination of malignant glioma patients with peptide-pulsed dendritic cells elicits systemic cytotoxicity and intracranial T-cell infiltration. Cancer Res 2001; 61: 842-7.

[17] Rosenberg SA. A new era for cancer immunotherapy based on the genes that encode cancer antigens. Immunity 1999; 10: 281-7.

[18] Izumoto S, Tsuboi A, Oka Y, et al. Phase II clinical trial of Wilms tumor 1 peptide vaccination for patients with recurrent glioblastoma multiforme. J Neurosurg 2008; 108: 963-71.

[19] Yamanaka R. Cell- and peptide-based immunotherapeutic approaches for glioma. Trends Mol Med 2008; 14: 228-35.

[20] Iizuka Y, Kojima H, Kobata T, Kawase T, Kawakami Y, Toda M. Identification of a glioma antigen, GARC-1, using cytotoxic T lymphocytes induced by HSV cancer vaccine. Int J Cancer 2006; 118: 942-9.

[21] Ohashi Y, Ueda M, Kawase T, Kawakami Y, Toda M. Identification of an epigenetically silenced gene: RFX1, in human glioma cells using restriction landmark genomic scanning. Oncogene 2004; 23: 7772-9.

[22] Ueda R, Yoshida K, Kawase T, Kawakami Y, Toda M. Preferential expression and frequent $\mathrm{IgG}$ responses of a tumor antigen: SOX5, in glioma patients. Int J Cancer 2007; 120: 1704-11.

[23] Ueda R, Iizuka Y, Yoshida K, Kawase T, Kawakami Y, Toda M. Identification of a human glioma antigen: SOX6, recognized by patients' sera. Oncogene 2004; 23: 1420-7.

[24] Ueda R, Ohkusu-Tsukada K, Fusaki N, et al. Identification of HLA-A2- and A24-restricted T-cell epitopes derived from SOX6 expressed in glioma stem cells for immunotherapy. Int $\mathrm{J}$ Cancer 2010; 126 (4): 919-29.

[25] Okada H, Pollack IF, Lotze MT, et al. Gene therapy of malignant gliomas: a phase I study of IL-4-HSV-TK gene-modified autologous tumor to elicit an immune response. Hum Gene Ther 2000; 11: 637-53.

[26] Sobol RE, Fakhrai H, Shawler D, et al. Interleukin-2 gene therapy in a patient with glioblastoma. Gene Ther 1995; 2: 164-7. 